\title{
OS NÚMEROS MÁGICOS DE BALL E A SEQUÊNCIA DE FIBONACCI
}

\author{
Eudes Antonio Costa \\ Universidade Federal do Tocantins \\ Colegiado de Matemática - Arraias \\ eudes@uft.edu.br
}

\section{Resumo}

Seja $x_{n}$ um número com $n$ algarismos. Para $n \geq 2$, o número de $n$ algarismos obtido pela inversão da posição dos algarismos de $x_{n}$ é chamado de número reverso de $x_{n}$ e é indicado por $x_{n}^{\prime}$. Admita que $x_{n}>x_{n}^{\prime}$ e escreva o número mágico de Ball $B=\left(x_{n}-x_{n}^{\prime}\right)+$ $\left(x_{n}-x_{n}^{\prime}\right)^{\prime}$. Em [6], e de forma independente em [4], mostra-se que todo número de Ball $B$ é múltiplo de 99. Para cada $k \geq 0$ inteiro, considere $x_{2 k}$ (ou $x_{2 k+1}$ ) um número qualquer e $B(k)$ a quantidade de possíveis números mágicos de Ball, ou seja, correspondentes às quantidades de algarismos $2,4,6, \ldots, 2 k, \ldots$ (ou $3,5,7, \ldots, 2 k+1, \ldots$ ) temos associada a sequência $1,4,12, \ldots, B(k), \ldots$ formada pela quantidade de números da Ball. Em [4] mostra-se que para todo inteiro $k \geq 2$ a quantidade $B(k)$ está entre $B(k-1)$ e $3^{k-1}+B(k-1)$. Do trabalho [6] conclui-se que a quantidade $B(k)$ é uma soma de termos, de índice par, da sequencia de Fibonacci.

\section{Abstract}

Let $x_{n}$ be a number with $n$ digits. For $n \geq 2$, the number of $n$ digits added by reversing the position of the digits of $x_{n}$ is called the reverse number of $x_{n}$ and is indicated by $x_{n}^{\prime}$. Admit $x_{n}>x_{n}^{\prime}$ and write the magic number of Ball $B=\left(x_{n}-x_{n}^{\prime}\right)+\left(x_{n}-x_{n}^{\prime}\right)^{\prime}$. In [6], and independently in [4], it is shown that every number of Ball's number $B$ is a multiple of 99. For each entire $k \geq 0$, consider $x_{2 k}$ (or $x_{2 k+1}$ ) any number, and $B(k)$ the quantity of Ball's magic numbers, that is, corresponding to the numbers of $2,4,6, \ldots, 2 k, \ldots$ (or $3,5,7, \ldots, 2 k+1, \ldots)$ we have associated the sequence $1,4,12, \ldots, B(k), \ldots$ formed by the amount of Ball's numbers. In [4] it is shown that for every integer $k \geq 2$ the amount $B(k)$ is between $B(k-1)$ and $3^{k-1}+B(k-1)$. From the paper [6] it is concluded that the quantity $B(k)$ is a sum of terms, even index, of the Fibonacci sequence. 
Costa, E.

\section{$1 \quad$ Números Mágicos de Ball}

Nossa discussão aqui se preocupa exclusivamente com a aritmética na base decimal, $\operatorname{assim} x_{n}=a_{n-1} \ldots a_{0}$ indica um número com $n$ algarismos, em que $a_{n-1}, \ldots, a_{0}$ são números inteiros de 0 a 9 . Para $n \geq 2$, o número de $n$ algarismos obtido pela inversão da posição dos algarismos de $x_{n}$ é chamado de número reverso de $x_{n}$ e é indicado por $x_{n}^{\prime}, \log x_{n}^{\prime}=a_{0} \ldots a_{n-1}$.

Nestas notas, $x_{n}$ representa um número inteiro positivo com $n$ algarismos, $n \geq 2 \mathrm{e}$ $a_{n-1} \neq 0$ e $x_{n}^{\prime}$ é o número reverso de $x_{n}$. Considere o seguinte algoritmo

Algoritmo 1.1. : [1, 2] O número de mágico Ball B.

1. Considere um número $x_{n}$;

2. Escreva o número reverso $x_{n}^{\prime}$;

3. Encontre o valor absoluto da diferença entre esses números, representado por $y_{n}=\left|x_{n}-x_{n}^{\prime}\right|=b_{n-1} b_{n-2} \cdots b_{2} b_{1} b_{0}$ (que deve ser considerado como um número de $n$ algarismos, mesmo quando o algarismo $b_{n-1}, b_{n-2}, \ldots$ for zero.);

4. Escreva o número reverso $y_{n}^{\prime}$;

5. Escreva o número $B=y_{n}+y_{n}^{\prime}$.

Para qualquer $B \neq 0$, chamamos o número $B$ de mágico de Ball se for o resultado do Algoritmo (1.1). Quando $x_{n}>x_{n}^{\prime}$, de forma simplificada obtemos o número mágico de Ball fazendo $B=\left(x_{n}-x_{n}^{\prime}\right)+\left(x_{n}-x_{n}^{\prime}\right)^{\prime}$.

Exemplo 1.2. [4] Para $n=3$, dado $x_{3}=843$, usamos o algoritmo para obter $x_{3}^{\prime}=348$, donde obtemos $y_{3}=843-348=495$. Assim, $y_{3}^{\prime}=594$. Finalmente, resulta que $B=495+594=1089$.

Para qualquer $x_{3}=a_{2} a_{1} a_{0}$, com $a_{2} \neq a_{0}$, como no Exemplo (1.2), o resultado final sempre será o número 1089. O mesmo fato ocorre quando temos um número inicial $x_{2}=a_{1} a_{0}$ com $a_{1} \neq a_{0}$, sempre obteremos o número 99 no final. Portanto, 99 e 1089 são exemplos de números de mágicos de Ball. Em [2, p. 8] (ou [1, p. 48]) prova-se que 1089 é o número resultante para todo o número $x_{3}$, como no Exemplo 1.2.

Em [2], o autor apresenta uma justificativa para o Algoritmo (1.1) retornar o número 1089 quando $n=3$, e independentemente em [3]. Na verdade, em [2] observa-se que o Algoritmo (1.1) resulta em $9 \times 11^{2}$, ou seja, 1089. Enquanto em [4, 6] propõem o Algoritmo (1.1) para todo número inteiro positivo $x_{n}$ para quaisquer $n \geq 2$.

A Tabela 1 apresenta a lista de números de Ball ao aplicar o Algoritmo (1.1) aos números $x_{n}$ com $n=2,4$ ou 6 algarismos. 
Costa, E.

\begin{tabular}{|c|c|c|c|c|}
\hline$n$ & número & condição & Número mágico de Ball & Quantidade \\
\hline$\overline{22}$ & $x=a_{1} a_{0}$ & $a_{1}>a_{0}$ & 99 & $\overline{1} 1$ \\
\hline 4 & $x=a_{3} a_{2} a_{1} a_{0}$ & $\begin{array}{l}a_{3}=a_{0} \text { e } a_{2}>a_{1} \\
a_{3}>a_{0} \text { e } a_{2}<a_{1} \\
a_{3}>a_{0} \text { e } a_{2}>a_{1} \\
a_{3}>a_{0} \text { e } a_{2}=a_{1}\end{array}$ & $\begin{array}{c}990 \\
9999 \\
10890 \\
10989\end{array}$ & 4 \\
\hline 6 & $x=a_{5} a_{4} a_{3} a_{2} a_{1} a_{0}$ & $\begin{array}{l}a_{5}=a_{0} \text { e } a_{4}>a_{1} \text { e } a_{3}<a_{2} \\
a_{5}=a_{0} \text { e } a_{4}>a_{1} \text { e } a_{3}>a_{2} \\
a_{5}=a_{0} \text { e } a_{4}>a_{1} \text { e } a_{3}=a_{2} \\
a_{5}=a_{0} \text { e } a_{4}=a_{1} \text { e } a_{3}>a_{2} \\
a_{5}>a_{0} \text { e } a_{4}>a_{1} \text { e } a_{3}<a_{2} \\
a_{5}>a_{0} \text { e } a_{4}>a_{1} \text { e } a_{3}>a_{2} \\
a_{5}>a_{0} \text { e } a_{4}>a_{1} \text { e } a_{3}=a_{2} \\
a_{5}>a_{0} \text { e } a_{4}<a_{1} \text { e } a_{3}>a_{2} \\
a_{5}>a_{0} \text { e } a_{4}<a_{1} \text { e } a_{3}<a_{2} \\
a_{5}>a_{0} \text { e } a_{4}<a_{1} \text { e } a_{3}=a_{2} \\
a_{5}>a_{0} \text { e } a_{4}=a_{1} \text { e } a_{3}=a_{2}\end{array}$ & $\begin{array}{c}99990 \\
108900 \\
109890 \\
9900 \\
1089990 \\
1098900 \\
1099890 \\
999999 \\
991089 \\
990099 \\
1099989\end{array}$ & 12 \\
\hline
\end{tabular}

Tabela 1: Exemplos extraídos de [4]

\section{Propriedades dos Números de Ball}

Consideramos números $x_{n}$, com $n \geq 2$. O resultado seguinte estabelece condições sobre a existência do número mágico de Ball $B$.

Proposição 2.1. [4, Afirmação 1, 2] (a) Considere $x_{n}$ para n par, isto é,

$$
x_{n}=a_{2 k-1} a_{2 k-2} \ldots a_{k+1} a_{k} a_{k-1} a_{k-2} \ldots a_{1} a_{0} .
$$

O número mágico de Ball B existe se uma das condições ocorre:

$$
a_{2 k-1} \neq a_{0} \text { ou } a_{2 k-2} \neq a_{1} \text { ou } \ldots \text { ou } a_{k+1} \neq a_{k-2} \text { ou } a_{k} \neq a_{k-1} \text {. }
$$

(b) Considere $x_{n}$ para $n$ impar, isto é,

$$
x_{n}=a_{2 k} a_{2 k-1} \ldots a_{k+2} a_{k+1} a_{k} a_{k-1} a_{k-2} \ldots a_{1} a_{0}
$$

ReviSeM, Ano 2021, $N^{\circ}$. 1, 19-25 
Costa, E.

O número mágico de Ball B existe se uma das condições ocorre:

$$
a_{2 k} \neq a_{0} \text { ou } a_{2 k-1} \neq a_{1} \text { ou } \ldots \text { ou } a_{k+2} \neq a_{k-2} \text { ou } a_{k+1} \neq a_{k-1} \text {. }
$$

A demonstração deste fato pode ser obtida em [4]. De acordo com a Proposição 2.1 , consideramos o número $x_{2 k}(k \geq 1)$, que consiste em um número par de algarismos (ou $x_{2 k+1}$ que consiste em um número ímpar número de algarismos) e, para cada $k$, $B(k)$ associa a quantidade de números mágicos de Ball B. Observando a Tabela 1, e outros exemplos, temos correspondente às quantidades iniciais de algarismos forem $2,4,6, \ldots, 2 k, \ldots$ (ou $3,5,7, \ldots, 2 k+1, \ldots)$, temos $1,4,12, \ldots, B(k), \ldots$ a sequência de quantidade de números mágicos de Ball. Veja que

$$
1 ; 4=1+3 ; 12=1+3+8 ; \ldots
$$

Nosso intento é formalizar o resultado sobre a quantidade de números mágicos de Ball associado ao número $x_{n} \operatorname{com} n=2 k$ algarismos (ou $n=2 k+1$ algarismos), e expressar $B(k)$ como a soma de termos (de índices pares) da sequencia de Fibonacci,

$$
1,1,2,3,5,8,13,21,34,55,89,144, \ldots
$$

isto é, a sequencia $F_{j}$ dada pela recorrencia $F_{j+2}=F_{j+1}+F_{j}$ para todo $j \geq 1$, veja [5].

Teorema 2.2. Para um número natural $x_{2 k}$ com $2 k$ algarismos, para todo $k \geq 1$, a quantidade de números mágicos de Ball $B(k)$ é $F_{2 k}+F_{2(k-1)}+\cdots+F_{2}$, em que $F_{j}$ éo termo de posição j da sequencia de Fibonacci (2.1).

Apresentaremos uma demonstração do Teorema 2.2 na Seção 4. Outro resultado interessante acerca dos números mágicos de Ball é o seguinte.

Teorema 2.3. [4, Afirmação 3], [6, Theorem 1] Todo número mágico de Ball B é um múltiplo do número 99.

Na próxima seção apresentaremos uma demonstração adaptada de [6].

\section{Demonstração do Teorema 2.3}

Antes da demonstração, vamos associar a cada número com $n$ algarismos $x_{n}=a_{n_{1}} \ldots a_{0}$, o número $X_{n}$ chamado de código de $x_{n}$. Este código $X_{n}$ compreende uma sequência de $0 s$ e $1 s$, tem algarismo final 0 e codifica as informações necessárias para passar do número inicial $x_{n}$ para o número de Ball $B$ resultante. Vamos apresentar a construção do $X_{n}$ informalmente. Escreva o número $x_{n}=a_{n-1} \ldots a_{0}$, o qual tomamos $a_{n-1}>a_{0}$, e abaixo dele, seu reverso $x_{n}^{\prime}=a_{0} \ldots a_{n-1}$, como mostrado abaixo: 
Costa, E.

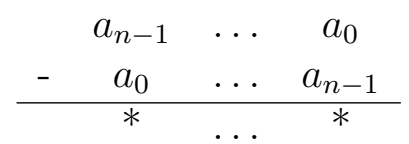

Considere o papel desempenhado pela $i$-ésima coluna da direita para esquerda $(i=$ $0, \ldots, n-1)$ na subtração do número $x_{n}^{\prime}$ de $x_{n}$. Defina o algarismo $z_{i}$ da seguinte maneira: se um dez (10) tiver que ser deslocado da $i+1$-ésima para a $i$-ésima coluna, $z_{i}$ será 1 ; caso contrário, é 0 . Dessa maneira, obtemos a sequencia $z_{0}, \ldots, z_{n-1}$ de $0 s$ e $1 s$. O número $z_{0} \ldots z_{n-1} \operatorname{com} n$ algarismos é chamado de código de $x_{n}$ e é denotado por $X_{n}$, ou seja, $X_{n}=z_{0} \ldots z_{n-1}$. Desde que estamos assumindo que $a_{n-1}>a_{0}, z_{0}=1$ e $z_{n-1}=0$. O número do $n-1$ algarismos obtido de $X_{n}$ pela exclusão do $z_{n-1}=0$ no final é denotado por $X_{\bar{n}}=z_{0} \ldots z_{n-2}$ e é chamado de código truncado de $x_{n}$.

Para ilustrar essas idéias, considere o número de seis algarismos $x_{6}=397862$. Subtraindo $x_{6}^{\prime}=268793$ de $x_{6}$, temos

\begin{tabular}{rrrrrr}
3 & $\not 9^{8}$ & $\not 1^{17}$ & $\not \not^{7}$ & $\not 6^{15}$ & $\not 2^{12}$ \\
$-\quad 2$ & 6 & 8 & 7 & 9 & 3 \\
\hline 1 & 2 & 9 & 0 & 6 & 9 \\
\hline \hline 0 & 0 & 1 & 0 & 1 & 1
\end{tabular}

As colunas nas quais um dez (10) foi deslocado da coluna adjacente à esquerda são (iniciando à direita) 0,1 e 3 , usando a notação acima temos

$$
z_{0}=1, z_{1}=1, z_{2}=0, z_{3}=1, z_{4}=0 \text { e } z_{5}=0 .
$$

Portanto temos o código $X_{6}=110100$ e o código trucado $X_{\overline{6}}=11010$. Conforme Tabela 1, para esse $x_{6}=397862$ em particular, temos o número de Ball

$$
B=129069+960921=1089990=99 \times 11010=99 \times X_{\overline{6}} .
$$

Considere $x_{n+1}$ um número com $n+1$ algarismos e $D$ denota o número 10, assim $x_{n+1}=a_{n} \ldots a_{0}=\sum_{i=0}^{n} a_{i} D^{i}$, com $a_{n}>a_{0}$ e $x_{n+1}^{\prime}=a_{0} \ldots a_{n}=\sum_{i=0}^{n} a_{n-i} D^{i}$. Usando desta notação, faremos a demonstração do Teorema 2.3 .

\section{Demonstração. do Teorema 2.3}

Sejam o número $x_{n+1}=\sum_{i=0}^{n} a_{i} D^{i}$ com $n+1$ algarismos, o número reverso $x_{n+1}^{\prime}=$ $\sum_{i=0}^{n} a_{n-i} D^{i}$ e o código $X_{n+1}=z_{0} \ldots z_{n}$ associado a $x_{n+1}$. Sem perda de generalidade, admita que $x_{n+1}>x_{n+1}^{\prime}$. Assim pela definição de $z_{0}, \ldots, z_{n}$, temos que

$$
x_{n+1}-x_{n+1}^{\prime}=\sum_{i=0}^{n}\left(a_{i}+z_{i} D-a_{n-i}-z_{i-1}\right) D^{i},
$$


Costa, E.

em que fazemos $z_{-1}=0$. Como $z_{n}=0$, obtemos

$$
\begin{aligned}
B & =\left(x_{n+1}-x_{n+1}^{\prime}\right)+\left(x_{n+1}-x_{n+1}^{\prime}\right)^{\prime} \\
& =\left(\sum_{i=0}^{n}\left(a_{i}+z_{i} D-a_{n-i}-z_{i-1}\right) D^{i}\right)+\left(\sum_{i=0}^{n}\left(a_{n-i}+z_{n-i} D-a_{i}-z_{n-i-1}\right) D^{i}\right) \\
& =\sum_{i=0}^{n}\left(a_{i}+z_{i} D-a_{n-i}-z_{i-1}+a_{n-i}+z_{n-i} D-a_{i}-z_{n-i-1}\right) D^{i} \\
& =\sum_{i=0}^{n}\left(z_{i} D-z_{i-1}+z_{n-i} D-z_{n-i-1}\right) D^{i} \\
& =\sum_{i=0}^{n} z_{i} D^{i+1}-\sum_{i=0}^{n} z_{i-1} D^{i}+\sum_{i=0}^{n} z_{n-i} D^{i+1}-\sum_{i=0}^{n} z_{n-i-1} D^{i} \\
z_{n}=z_{-1}=0 & D^{2} \sum_{i=1}^{n} z_{n-i} D^{i-1}-\sum_{i=1}^{n} z_{n-i} D^{i-1} \\
= & \left(D^{2}-1\right) z_{0} \cdots z_{n-1}=99 \times X_{\overline{n+1}} .
\end{aligned}
$$

\section{Demonstração do Teorema 2.2}

O Teorema 2.3 mostra que o número $B(k)$ que buscamos é o mesmo que o número de diferentes códigos truncados $X_{\overline{2 k}}$ ou, equivalentemente, códigos $X_{2 k}$. Inicialmente em [6], para determinar a quantidade de códigos, o autor considerou apenas o caso em que $a_{n}$ é (sempre) maior que $a_{0}$, ao considerar $x_{n+1}$ maior que $x_{n+1}^{\prime}$, e obteve o seguinte resultado

Proposição 4.1. [6, Theorem 3] Para um número natural $x_{2 k}$ com $2 k$ algarismos, com $k \geq 1$ tem-se que a quantidade $B(k)$ de números de Ball é igual a $F_{2 k}$, em que $F_{j}$ é o termo de posição $j$ da sequencia de Fibonacci (2.1) .

A demonstração pode ser consultada em [6]. Um resultado auxiliar para demonstração do Teorema 2.2 é o seguinte.

Proposição 4.2. Se $B_{0}$ é um número de Ball associado a um número $x_{n}$ com $n$ algarismos então $10 B_{0}$ é um número de Ball associado a um número $x_{n+2}$ com $n+2$ algarismos. 
Costa, E.

Demonstração. (O resultado e a demonstração podem ser inferidos de $[6$, Conclundig remarks] .) Dado um número $x_{n}=a_{n-1} \ldots a_{0}$ com $n$ algarismos, pelo Teorema 2.3 temos que o número de Ball $B_{0}$ associado ao número $x_{n}$ é $99 \times X_{\bar{n}}$, em que $X_{\bar{n}}$ é o código truncado associado ano número $x_{n}$. E mais ao número $x_{n}$ temos associado o código $X_{n}=z_{n} \cdots z_{0}$, ao acrescentar um mesmo algarismo $a$ no início e no fim do número $x_{n}$ obtemos $x_{n+2}=a a_{n} \ldots a_{0} a$ um número com $n+2$ algarismos e obtemos o código $X_{n+2}=0 z_{n} \cdots z_{0} 0$. Novamente, pelo Teorema 2.3 temos que o número de Ball associado ao número $x_{n+2}$ é $99 \times X_{\overline{n+2}}=99 \times 10 \times X_{\bar{n}}=10 B_{0}$.

Demonstração. do Teorema 2.2

Dado número $x_{2 k} \operatorname{com} n=2 k$ algarismos, suponha que $a_{n-1}>a_{0}$, segue da Proposição (4.1) que $B(k)=F_{2 k}$. Agora, no caso em que $a_{n-1}=a_{0}$, segue da Proposição (4.2) que a quantidade de números de Ball é a mesma para $n-2$ algarismos, assim temos $B(k-1)=F_{2(k-1)}$. Recursivamente, obtemos o resultado.

\section{Referências}

[1] J. Ball, Think of a Number, Dorling Kinderly Limited, Great Britain, 2005.

[2] W. W. R. Ball, Mathematical Recreations and Essays, The Macmillan Company, New York, (Tenth Edition), 1926.

[3] E. A. Costa, Mais um Número Mágico, Revista do Professor de Matemática 80, 2013, 23-24 .

[4] E. A Costa e E. G. Mesquita, O Número Mágico M, Revista da Olimpíada do IME-UFG 9, 2014, 33-43. $<<$ https://files.cercomp.ufg.br/weby/up/1170/o/Eudes9.pdf $>>$

[5] N. N. Vorobev, Numeros de Fibonacci. Lecciones populares de matemáticas: Editorial MIR, Moscú, Rússia, 1974.

[6] R. Webster, A combinatorial problem with a Fibonacci solution, Fibonacci Quart. 33 (1995), no. 1, 26-31.

Submetido em 17 de Julho de 2020. Aceito em 30 de Setembro de 2020. 